\title{
COVID-19: Current Trends in Invitro Diagnostics
}

\author{
R. Arun Krishnan ${ }^{1}$ (D) Rhema Elizabeth Thomas ${ }^{1} \cdot$ Ajaikumar Sukumaran $^{1}$. \\ Jofy K. Paul ${ }^{1}$ - D. M. Vasudevan ${ }^{1}$
}

Received: 4 May 2020/Accepted: 15 June 2020/Published online: 27 June 2020

(C) Association of Clinical Biochemists of India 2020

\begin{abstract}
The novel coronavirus SARS-CoV-2 is the seventh known species of coronavirus, infectious to human beings. The pandemic COVID-19 spread all over the world with an unprecedented spreading rate after its first appearance in Wuhan, China. As a novel viral disease there in no antiviral treatment or vaccine for the COVID-19. At present, the early detection and the quarantine of infected patients are the ways to stop the spreading of the disease. This review will discuss about the current invitro diagnostic methods used worldwide for the early and accurate diagnosis of COVID-19. Currently the nucleic acid based polymerase chain reaction is used as the reliable diagnostic platform and antigen/antibody detection immunoassays are playing the role of screening tests for early detection and prognosis in COVID-19 treatment.
\end{abstract}

Keywords SARS-CoV-2 - Reverse transcriptase PCR . Loop mediated isothermal amplification - Lateral flow immunoassay · ELISA · CLIA

\section{Introduction}

The Coronavirus disease 2019 (COVID-19) is the latest pandemic gaoling the humanity, having very high spreading rate and approximately 5-6\% of mortality worldwide. This novel beta coronavirus is an enveloped non-segmented positive sense RNA virus. The Severe Acute Respiratory Syndrome Coronavirus-2 (SARS-CoV-2) genome structure comprises single stranded RNA with nucleocapsid protein which are enclosed by membrane proteins, envelope proteins and spike glycoproteins [1] (Fig. 1). As a coronavirus, the SARS-CoV-2 has high similarity with other viruses like SARS-CoV and MERS-CoV [2]. The entry of the novel coronavirus to host is through the interaction between the densely glycosylated spike proteins to the receptors on host cell membrane. The spike glycoprotein is a trimeric class I fusion protein consisting S1 and $\mathrm{S} 2$ domains [3]. It is reported that there is $55 \%$ similarity in $\mathrm{S} 1$ domain of spike glycoproteins among SARS-CoV-2 and SARS-CoV and $91 \%$ similarity in case of S2 domain. It is evident that the SARS-CoV-2 can infect the human respiratory epithelial cells through interaction of spike protein with the human ACE2 receptor. [4].

The coronaviruses are large RNA viruses $(65-125 \mathrm{~nm}$ in diameter) come under the Coronaviridae family in the Nidovirales order. Normally the coronavirus genome contains six open reading frame (ORFs) which encodes for the structural as well as accessory proteins in the virus. The nucleocapsid protein (N-protein) in the coronavirus binds to RNA genome and forms a capsid around the enclosed nucleic acid. The function of $\mathrm{N}$-protein includes the interaction with membrane protein during viral assembly, assists in RNA synthesis/folding and affects host cell responses, including cell cycle and translation. The structural and accessory proteins are essential for viral replication, genome maintenance and pathogenesis of the virus. [5].
R. Arun Krishnan

arunkvm123@gmail.com

1 R\&D Reagent Department, Agappe Diagnostics Limited, Cochin, Kerala, India 
Fig. 1 Schematic diagram of SARS-COV-2

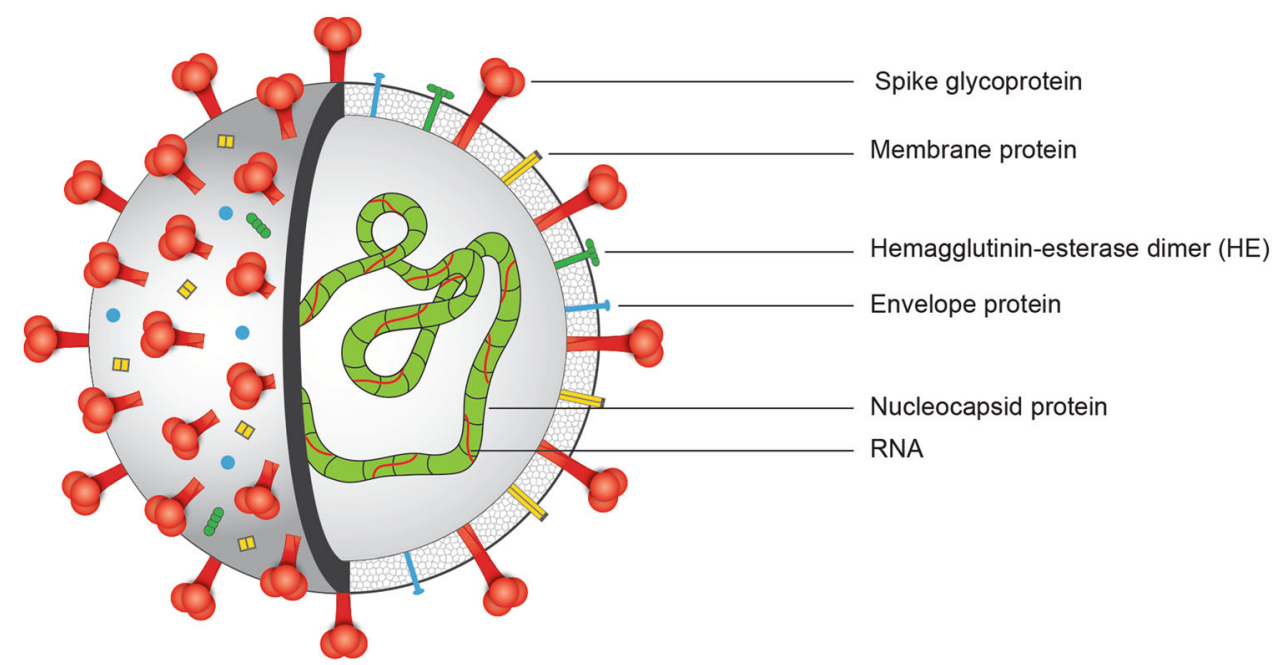

\section{Current Molecular Diagnostic Techniques for COVID-19}

The currently available invitro diagnostic techniques can be broadly classified into (1) Nucleic acid based assays and (2) Serological assays. These are described below.

\section{Nucleic Acid Based Assays}

At present the most widely used approved test for the diagnosis of COVID-19 is the Polymerase Chain Reaction (PCR). Two different strategies are in use for PCR based assays; the Reverse Transcriptase PCR (RT-PCR) and the Loop Mediated Isothermal Amplification PCR (LAMP PCR). Both methods offer high sensitivity (85-90\%) and specificity for the COVID-19 diagnosis as the methods are focusing on direct amplification of the virus genetic material. The RT-PCR is quantitative in nature whereas the LAMP PCR is qualitative. As compared to RT-PCR, LAMP PCR will be more cost effective and less time consuming. High throughput screening is another nucleic acid detection technology. It is costly and has high equipment dependency making it less widely used. Even though the aforementioned methods can offer nearly $90 \%$ accurate result, the improper sample collection, handling and transportation may lead to false negative results, obviously decreasing the sensitivity of the assay.

\section{Reverse Transcriptase PCR (RT-PCR)}

The RT-PCR is the most common and effective method used in the market to detect SARS-CoV-2. In RT-PCR, reverse transcriptase converts virus RNA into cDNA following amplification into millions of copies of DNA using a set of specific primers and probes. The amplification taking place in 3 steps: [1] denaturation [2] annealing and
[3] elongation. These three steps take place at $95{ }^{\circ} \mathrm{C}$ for $30 \mathrm{~s}, 50^{\circ}$ for $30 \mathrm{~s}$ and $72{ }^{\circ} \mathrm{C}$ for $60 \mathrm{~s}$ respectively. The primers target and amplify different regions for SARS$\mathrm{CoV}-2$ such as nucleocapsid protein (N) gene, envelope protein (E) gene and ORF1ab gene regions which can be determined within the same cycle and separately for confirmatory testing [6]. The turnaround time for sample analyses is 2.5-3.5 h. One-step RT-PCR assay to detect E gene and RNA-dependent RNA polymerase (RdRp gene) regions of SARS-Cov-2 has been developed by Tib-Molbiol [7]. Predominantly, upper respiratory samples including nasopharyngeal swabs and oropharyngeal swabs are recommended for analysis. There are many breakthrough assays developed by various IVD manufacturers including Abbott, Bosch and Cepheid where a specific gene of SARS-CoV-2 is detected within few minutes. Although RT-PCR is the most widely used confirmatory test for COVID-19, this technique is not robust and can only be performed by skilled analysts due to various biological safety hazards making sample detection cumbersome in many cases [8].

Even though the RT-PCR is a robust technology there are many factors contributing to false-positive and falsenegative results. Mutations in primers targeting virus genome can lead to false negative results. Laboratory practices and safety procedures including collection, transportation and handling also contribute to false negative results [9]. The sampling timing and optimum sample types play significant role in obtaining highly sensitive and highly specific results [10]. In most PCR assays two different regions of genes are targeted. Hence the result interpretation will be positive, negative or inconclusive. If both genes are amplified the result is positive; If only one gene is positive, that is called inconclusive; and negative could be a false negative. So, inconclusive and negative persons should be treated as doubtful. 


\section{Loop Mediated Isothermal Amplification (LAMP)}

The efforts on minimizing length of the entire PCR process and practice of isothermal amplification has resulted in the development of loop-mediated isothermal amplification. The LAMP is a highly specific isothermal amplification technique with an analytical limit of detection of 75 copies per $\mu$ l. In LAMP, conversion of RNA to DNA is followed by amplification of sample genome using 4-6 primers (forward inner primer, forward outer primer, reverse inner primer and reverse outer primer) targeting different regions of the DNA [11]. The LAMP has several advantages over RT-PCR. It is considered to be more user friendly with easy detection, speed and less background signal when compared to RT-PCR. In addition to its speed, LAMP shows high level of specificity and high amplification efficiency than RT-PCR [12]. The recently developed Chitra Gene LAMP-N by Sree Chitra Thirunal Medical Institute and Technology in collaboration with Agappe Diagnostics Ltd. detects two different regions of $\mathrm{N}$ gene. The turnaround time of $20 \mathrm{~min}$ for the analysis of 30 samples is a remarkable achievement. However, stringent evaluations on commercial LAMP kits are needed for accuracy check and processing of large samples is an impetus for COVID-19 testing.

Apart from RT-PCR and LAMP, United States Centers for Disease Control and Prevention (CDC) approved onestep real time reverse transcriptase polymerase chain reaction (rRT-PCR) to quantitatively detect viral particles in more than 90 samples within $45 \mathrm{~min}$ [13]. Recently, Specific High Sensitivity Enzymatic Reporter Unlocking (SHERLOCK) that allows multiplexed, portable, and ultrasensitive detection of RNA is a detection strategy used to combat SARS-CoV-2. Cas13a ribonucleases used for RNA sensing binds to amplified RNA which when activated, cleaves fluorophore-quencher probes emitting fluorescence. SHERLOCK has previously been used to detect clinical samples for Zika Virus [14]. Nevertheless, there is a need for further optimisation of this technique in larger samples to achieve large scale evaluation of SARS-CoV-2.

\section{Serological Assays}

Though the rRT-PCR is considered as the 'Gold Standard' in the clinical diagnosis of COVID-19, it has own limitations. As a pandemic, screening and isolation of the infected patients is the only way to reduce the spread of COVID-19. In RT-PCR, the testing directly look for the presence of viral DNA but serological tests detect the presence of antibodies/antigens in human blood against the COVID-19. Hence RT-PCR will give the positive results in 3-7 days of infection but serological test will take $7-14$ days to give positive results. The test cost and testing time of PCR based assays inevitably demands the development of rapid serology tests [15-17]. Several immunoassays are developed by various IVD companies for detecting COVID-19 infection in serum, plasma and whole blood. Among these strategies Lateral flow immunoassays, ELISA and Chemiluminescence are most promising approaches.

The limitation of rRT-PCR to detect COVID-19 past infection and the progress of the disease, increases the importance of serological assays. The serological tests can detect both active and past infections, if the tests are performed within the correct time frame after the onset of the disease. In case of antibody detection assay, both IgG and IgM antibodies against the COVID-19 are the target analytes where IgM appears in blood within a week of time and IgG expression will take more than 10 days. Apart from antibody detection, antigen detection against spike glycoprotein and nucleocapsid protein is also under development which offers early detection of COVID-19 infection. In case of RT-PCR one of the important reasons for false negative results is improper sampling (throat swab) and transport, whereas the sample of choice for serological test is serum/plasma/whole blood which are easy to handle and transport. Serological tests like LFIA for antigen/antibody detection can be used as a rapid test or a point of care testing which offers quick screening of the patients with limited expertise [18]. Detecting antibody titre is important for convalescent plasma therapy and the development of vaccines for COVID-19. Apart from that, serological testing would also provide valuable information regarding the course and degree of immune response as well as the durability of immunity in both infected individuals and participants in the vaccine clinical trials. [19].

\section{Lateral Flow Immunoassay (LFIA)}

The LFIA can be utilized as a preliminary testing tool for COVID-19 in case of community spread or in mass population screening. At present the COVID-19 $\mathrm{IgG} / \mathrm{IgM}$ antibody evaluation kits based on LFIA is widely available in market. Upon COVID-19 infection the IgM and IgG antibodies will start to develop in our body from 7-10 days and 14-20 days respectively. Though IgM can be detected earlier it also decreases and disappears earlier but IgG can persist for a long time after the infection. Even though the antibody test kits are reliable for preliminary screening, the test cannot be used as a confirmatory test because of nonspecific interaction of antibodies and other proteins in the blood with capture and detector molecules in the membrane, which may results in false positive or false negative results [20].

The main advantages of LFIA are that it can be used as a rapid POC test and it can be detected using finger-pricked 
blood or serum/plasma. It does not require any instruments or expertise staff for carrying out the test and it gives result in 5-30 min [21]. The LFIA antibody test for COVID-19 can also be considered as a prognostic tool there by provides information about the immune status of the patient. Mostly the LFIA results are qualitative but can be made quantitative by the use of customized color readers. Currently COVID-19 antigen LFIA test is under development which will offer more sensitive and specific result for COVID-19 diagnosis and will detect the viral antigen in 3 days of infection [22].

\section{Enzyme Linked Immunosorbent Assay (ELISA) and Chemiluminescence Immunoassay (CLIA)}

The ELISA and CLIA platforms are widely using serology techniques for the quantitative detection of specific antigen or antibody in the samples. Zhang et al. have developed an enzyme linked immunosorbent assay for the detection of COVID-19 IgM and IgG antibody from serum sample. SARS-CoV-2 Rp3 nucleocapsid protein is adsorbed on the surface of a 96 well plate as capture molecule and anti-IgG antibody conjugated with horse radish peroxidase is used as the detector molecule [23]. Manual ELISA kits for detecting COVID-19 have been developed by IVD manufactures like IBL International, DRG Diagnostics $\mathrm{GmbH}$, Epitope Diagnostics and Euroimmun for detecting the $\mathrm{IgG}$ and IgM antibodies developed in response to COVID-19 infection [24]. ELISA and CLIA based COVID-19 antigen detection kits are under development by IVD manufacturers worldwide.

Automated CLIA method can also detect IgG and IgM antibody in serum or plasma. The advantages of automated CLIA analyzers compared to rapid LFIA tests is the very high throughput of samples and the ability to detect other biomarkers like C- reactive protein simultaneously [22]. DZ-Lite SARS-CoV-2 CLIA developed by Diazyme, USA and MAGLUMI CLIA developed by Snibe, China are chemiluminescent analyzers which can detect IgG and IgM antibodies of COVID-19.

\section{Non-specific Tests for COVID-19}

Besides the aforementioned specific tests, certain routine biochemical and haematological tests show abnormal results in COVID 19 patients. Upon COVID-19 infection the level of prothrombin time, lactate dehydrogenase (LDH), D-dimer, alanine transaminase (ALT), C-reactive protein $(\mathrm{CRP})$, erythrocyte sedimentation rate (ESR) and creatine kinase are elevated [25]. There is a marked lymphopenia with depletion of CD4 and CD8 lymphocytes in the early phase of the disease. As the disease progress patients show higher levels of interleukin-2 (IL-2), IL-7,
IL-10, granulocyte colony-stimulating factor(GCSF), interferon gamma-induced protein, monocyte chemotactic protein 1 (MCP1), macrophage inflammatory protein alpha and tumour necrosis factor- $\alpha$ (TNF- $\alpha)$. It's also noted that patients in intensive care show significant increase in amylase and D-dimer. And in case of non survivors there is a marked elevation in the level of ferritin, blood urea, neutrophil count, and D-dimer and creatinine levels. But procalcitonin (PCT) is not elevated in COVID 19. So Procalcitonin level can be used for the differential diagnosis of COVID 19 from bacterial pneumonia [26, 27].

The level of CRP starts to rise at the time of mild pneumonia and reaches peak at severe pneumonia. The CRP is also used as a marker of inflammation and the rise correlates with the level of inflammation regardless of the factors such as age, sex and physical condition. It is reported that the CRP levels are positively correlated with lung lesions in early stage of COVID 19 cases and can be used as a key indicator for disease monitoring [28, 29].

\section{Conclusion}

The nucleic acid based diagnostics methods and serological assays described above are serving as strong diagnostics tools for the COVID-19 diagnosis. The assurance of quality of developed diagnostics kits is having prime importance at this scenario. Even though recently many developments happened in the field of serological diagnosis for COVID19 , still there are many concerns regarding the sensitivity and specificity of the assays. The complexity, cost effectiveness and limitations of nucleic acid based diagnostic tools, impetus the innovative development of well standardized, high sensitive, specific and low cost serological assays for COVID-19 diagnosis.

\section{Compliance with Ethical Standards}

Conflict of interest All authors declare that there is no conflict of interest.

\section{References}

1. Huang C, Wang Y, Li X, Ren L, Zhao J, Hu Y, et al. Clinical features of patients infected with 2019 novel coronavirus in Wuhan, China. Lancet. 2020;395(10223):497-506.

2. Zhu N, Zhang D, Wang W, Li X, Yang B, Song J, et al. A novel coronavirus from patients with pneumonia in China, 2019. N Engl J Med. 2020;382(8):727-33.

3. Wrapp D, Wang N, Corbett K, Goldsmith J, Hsieh C, Abiona O, et al. Cryo-EM structure of the 2019-nCoV spike in the prefusion conformation. Science. 2020;367(6483):1260-3.

4. Vankadari N, Wilce J. Emerging COVID-19 coronavirus: glycan shield and structure prediction of spike glycoprotein and its 
interaction with human CD26. Emerg Microb Infect. 2020;9(1):601-4.

5. Chen Y, Liu Q, Guo D. Emerging coronaviruses: genome structure, replication, and pathogenesis. J Med Virol. 2020;92(4):418-23.

6. Chu D, Pan Y, Cheng S, Hui K, Krishnan P, Liu Y, et al. Molecular diagnosis of a novel coronavirus (2019-nCoV) causing an outbreak of pneumonia. Clin Chem. 2020;66(4):549-55.

7. Vashist S. In vitro diagnostic assays for COVID-19: recent advances and emerging trends. Diagnostics. 2020;10(4):202.

8. WHO int. 2020. Laboratory testing for 2019 novel coronavirus (2019-Ncov) in suspected human cases. WHO/COVID-19/laboratory/2020.5.

9. Tahamtan A, Ardebili A. Real-time RT-PCR in COVID-19 detection: issues affecting the results. Expert Rev Mol Diagn. 2020;20(5):453-4.

10. Kim J, Ko J, Kim Y, Kim Y, Kim J, Chung Y, et al. Viral load kinetics of SARS-CoV-2 infection in first two patients in Korea. J Korean Med Sci. 2020;35(7):e86.

11. Notomi T. Loop-mediated isothermal amplification of DNA. Nucleic Acids Res. 2000;28(12):e63.

12. Zhang G, Brown E, González-Escalona N. Comparison of realtime PCR, reverse transcriptase real-time PCR, loop-mediated isothermal amplification, and the FDA conventional microbiological method for the detection of Salmonella spp. produce. Appl Environ Microbiol. 2011;77(18):6495-501.

13. Centers for Disease Control and Prevention. 2020. Coronavirus Disease 2019 (COVID-19).

14. Kellner M, Koob J, Gootenberg J, Abudayyeh O, Zhang F. SHERLOCK: nucleic acid detection with CRISPR nucleases. Nat Protoc. 2019;14(10):2986-3012.

15. Xie X, Zhong Z, Zhao W, Zheng C, Wang F, Liu J. Chest CT for typical 2019-nCoV pneumonia: relationship to negative RT-PCR testing. Radiology. 2020. https://doi.org/10.1148/radiol. 2020200343.

16. Li J, Ye G, Chen L, Wang J, Li Y. Analysis of false-negative results for 2019 novel coronavirus nucleic acid test and related countermeasures. Chin J Lab Med. 2020;43:E006.

17. Pan Y, Li X, Yang G, Fan J, Tang Y, Zhao J, et al. Serological immunochromatographic approach in diagnosis with SARS-CoV2 infected COVID-19 patients. J Infect. 2020. https://doi.org/10. 1101/2020.03.13.20035428v1.

18. Carter L, Garner L, Smoot J, Li Y, Zhou Q, Saveson C, et al. Assay techniques and test development for COVID-19 diagnosis. ACS Cent Sci. 2020;6(5):591-605.
19. Winter A, Hegde S. The important role of serology for COVID19 control. Lancet Infect Dis. 2020. https://doi.org/10.1016/ S1473-3099(20)30322-4.

20. Tan W, Lu Y, Zhang J, Wang J, Dan Y, Tan Z, et al. Viral kinetics and antibody responses in patients with COVID-19. medRxiv. 2020. https://doi.org/10.1101/2020.03.24.20042382v1.

21. Sukumaran A, Thomas T, Thomas R, Thomas R, Paul J, Vasudevan D. Development and troubleshooting in lateral flow immunochromatography assays. Indian J Clin Biochem. 2020. https://doi.org/10.1007/s12291-020-00887-5.

22. Xiang J, Yan M, Li H, Liu T, Lin C, Huang S, et al. Evaluation of enzyme-linked immunoassay and colloidal gold-immunochromatographic assay kit for detection of novel coronavirus (SARSCov-2) causing an outbreak of pneumonia (COVID-19). medRxiv. 2020. https://doi.org/10.1101/2020.02.27.20028787.

23. Zhang W, Du R, Li B, Zheng X, Yang X, Hu B, et al. Molecular and serological investigation of 2019-nCoV infected patients: implication of multiple shedding routes. Emerg Microb Infect. 2020;9(1):386-9.

24. Mungroo M, Khan N, Siddiqui R. Novel coronavirus: current understanding of clinical features, diagnosis, pathogenesis, and treatment options. Pathogens. 2020;9(4):297.

25. Wang Y, Wang Y, Chen Y, Qin Q. Unique epidemiological and clinical features of the emerging 2019 novel coronavirus pneumonia (COVID-19) implicate special control measures. J Med Virol. 2020;92(6):568-76.

26. Wang D, Hu B, Hu C, Zhu F, Liu X, Zhang J, et al. Clinical characteristics of 138 hospitalized patients with 2019 novel coronavirus-infected pneumonia in Wuhan, China. JAMA. 2020;323(11):1061.

27. Hassan S, Sheikh F, Jamal S, Ezeh J, Akhtar A. Coronavirus (COVID-19): a review of clinical features, diagnosis, and treatment. Cureus. 2020;12:e7355.

28. Chen N, Zhou M, Dong X, Qu J, Gong F, Han Y, et al. Epidemiological and clinical characteristics of 99 cases of 2019 novel coronavirus pneumonia in Wuhan, China: a descriptive study. Lancet. 2020;395(10223):507-13.

29. Wang L. C-reactive protein levels in the early stage of COVID19. Médecine et Maladies Infectieuses. 2020;50(4):332-4.

Publisher's Note Springer Nature remains neutral with regard to jurisdictional claims in published maps and institutional affiliations. 